\title{
Two cases of periodic fever syndrome with coexistent mevalonate kinase and Mediterranean fever gene mutations
}

\author{
Mustafa Çakan ${ }^{1}$, Nuray Aktay-Ayaz ${ }^{1}$, Gonca Keskindemirci², Şerife Gül Karadağ ${ }^{1}$ \\ Clinics of ${ }^{1}$ Pediatric Rheumatology, and ${ }^{2}$ Pediatrics, Kanuni Sultan Süleyman Research and Training Hospital, Istanbul, \\ Turkey.E-mail: mustafacakan@hotmail.com \\ Received: 28th January 2017, Revised: 5th April 2017, Accepted: 11th April 2017
}

\begin{abstract}
SUMMARY: Çakan M, Aktay-Ayaz N, Keskindemirci G, Karadağ ȘG. Two cases of periodic fever syndrome with coexistent mevalonate kinase and Mediterranean fever gene mutations. Turk J Pediatr 2017; 59: 467-470.

The periodic fever syndromes are autoinflammatory diseases that present with recurrent fever, serositis and rash. Familial Mediterranean fever is the most common periodic fever syndrome and characterized by recurrent attacks of fever, arthritis, peritonitis, pleuritis that typically last 1-3 days. Hyperimmunoglobulinemia D syndrome is another example of periodic fever syndromes and patients have recurrent fever attacks for 3-7 days accompanied by abdominal pain, rash, vomiting, diarrhea, arthralgia, arthritis, aphthous ulcers, and cervical lymphadenopathy. In some cases the clinical picture of the patient does not fit to one autoinflammatory syndrome because of the digenic inheritance. This may cause to overlap or atypical clinical features or an unexpected response to treatment. Herein we report two cases of hyperimmunoglobulinemia D syndrome that also had MEFV gene mutations and familial Mediterranean fever phenotype.
\end{abstract}

Key words: digenic inheritance, familial Mediterranean fever, hyperimmunoglobulinemia $D$ syndrome, periodic fever.

Periodic fever syndromes are a group of monogenic autoinflammatory disorders that are characterized by recurrent fever and systemic inflammation attacks ${ }^{1}$. Hyperimmunoglobulinemia D syndrome (HIDS) and familial Mediterranean fever (FMF) are the two most common examples of the periodic fever syndromes. HIDS, also known as partial mevalonate kinase deficiency or hyperimmunoglobulinemia $\mathrm{D}$ with periodic fever syndrome, is an autosomal recessive periodic fever syndrome. Fever attacks usually begin during the first year of life and last for 3-7 days. The attacks are separated by 1-2 month, symptom free intervals. During attacks besides fever; arthralgia, arthritis, abdominal pain, mouth ulcers, rash, and cervical lymphadenopathy may be seen. The disease is caused by mutations in the mevalonate kinase $(M V K)$ gene. There is no proven treatment of the disease, but colchicine and corticosteroids-on-demand are suggested as initial treatments. ${ }^{1,2}$

FMF is the prototype of the periodic fever syndromes and is caused by mutations in the Mediterranean fever (MEFV) gene. Fever and serositis attacks last 1-3 days and colchicine is the standard initial treatment for all cases. ${ }^{1,3}$ It is thought that having more than one periodic fever syndrome gene mutation may alter the disease phenotype and response to treatment ${ }^{1}$. Herein we report two cases of periodic fever syndrome with both $M V K$ gene and MEFV gene mutations.

\section{Case Reports}

\section{Case 1}

A 3-year-old boy was admitted to pediatric rheumatology outpatient clinic with the complaint of recurrent fever. The fever had started when he was 4 months old, not after the first vaccine injection but the second one. It was lasting for 3-7 days and repeating every 1-2 months. In the last 3 attacks the mother was also describing non-migrating urticarial rash and diarrhea. He was hospitalized for two times around 10 days because of the resistant fever without a focus and all the 
microbiological studies including cerebrospinal fluid examinations had not yielded any positive result. During febrile periods the patient had extremely high acute phase reactants; C-reactive protein (CRP) $234 \mathrm{mg} / \mathrm{L}$ (normal: 0-5 mg/L), serum amyloid A (SAA) $420 \mathrm{mg} / \mathrm{L}$ (normal 0-6.4 $\mathrm{mg} / \mathrm{L}$ ), erythrocyte sedimentation rate (ESR) $120 \mathrm{~mm} / \mathrm{h}$. Since FMF is common in our population, it was considered in the patient and MEFV gene analysis was studied that showed heterozygous R761H mutation and colchicine treatment was started. Under four months of colchicine treatment he had two more attacks and was referred to our clinic for consultation. The clinical picture of the patient was more suggestive of HIDS, because fever periods had started during infancy and some were lasting more than 3 days. Also diarrhea and urticaria-like rash was accompanying to the fever during the last attacks. On MVK gene analysis, compound heterozygous mutations (N205D and V377I) were found. The patient has been followed for 1 year and currently using colchicine daily and oral prednisolone during attacks. He had 3 attacks during this period that lasted 1 day with oral prednisolone and acute phase reactants were normal during attack free periods. With this current treatment as both the attack frequency was decreased and APRs were normal in between the attacks we did not consider biologic therapy (interleukin - 1 inhibitor) for this patient.

\section{Case 2}

A 3.5-year-old boy was admitted to emergency department with the complaints of fever, abdominal pain and convulsion. The patient had fever for 3 days and on physical examination he had hepatosplenomegaly and cervical lymphadenopathy. Medical history was remarkable for recurrent fever attacks that started around one year of age. Each attack was lasting for 3-7 days and was repeating every 1-3 months. He also had two simple febrile convulsions during previous attacks. There was consanguinity between parents and one cousin of the patient had FMF. He had anemia (hemoglobin $8.1 \mathrm{~g} / \mathrm{dl}$ ), thrombocytopenia (platelet $108,000 / \mathrm{mm}^{3}$ ) and high acute phase reactants (CRP $182 \mathrm{mg} / \mathrm{L}$, SAA $258 \mathrm{mg} / \mathrm{L}$, ESR $100 \mathrm{~mm} / \mathrm{h})$. We suspected concurrent infection and started broad-spectrum antibiotics after obtaining cultures but we could not demonstrate any viral or bacteriologic etiology. Neither malignant or hemophagocytic cells were observed on bone marrow examination. The fever lasted 3 more days and thrombocytopenia resolved after one week. $M E F V$ gene analysis showed heterozygous M680I mutation. Colchicine treatment was started with the diagnosis of FMF and during one year followup anemia and hepatosplenomegaly regressed and had two attacks. But later the patient had three more attacks with fever, urticariallike rash, abdominal pain, diarrhea, cervical lymphadenopathy and chest pain for 3-5 days during the next 6 months. HIDS was considered in differential diagnosis and $M V K$ gene analysis showed homozygous V377I mutation. Colchicine treatment was continued and oral prednisolone on-demand was added to the regimen. Fever attacks lasted around 1.5 days with prednisolone use but continued to have attacks every 1-2 months. Anakinra $(4 \mathrm{mg} / \mathrm{kg} /$ day, sc) treatment was initiated due to frequent attacks and moderately high acute phase reactants in between the attack-free periods. Anakinra was used for 9 months and switched to canakinumab (3 $\mathrm{mg} / \mathrm{kg} / \mathrm{month})$ that is being used for the last 3 months. He had only one attack in the last year, since starting anti-interleukin-1 treatment and acute phase reactants are within normal limits.

Informed consent was received from the families.

\section{Discussion}

The hereditary periodic fever syndromes are a group of monogenic disorders manifesting with recurrent fever and inflammation. ${ }^{1}$ The term autoinflammation was described first time in 1999 to denote an emerging family of clinical disorders characterized by episodes of seemingly unprovoked inflammation without high-titer autoantibodies or antigen-specific $\mathrm{T}$ lymphocytes. ${ }^{2}$ Monogenic autoinflammatory diseases are primarily inborn errors of innate immunity contrary to the autoimmune diseases which are primarily due to errors of the adaptive immunity ${ }^{1}$. There are many well-known diseases under the heading of monogenic autoinflammatory disorders and with better understanding of underlying pathophysiologic mechanisms and genetics, new phenotypes are being described.2,4

Familial Mediterranean fever and HIDS are the 
two most common examples of the hereditary periodic fever syndromes with common features of periodic fever, abdominal pain, arthralgia and arthritis. ${ }^{3}$ Fever lasting for 3-7 days and starting during infancy especially triggered by vaccinations, accompanying urticaria-like rash, diarrhea, aphthous stomatitis, cervical lymphadenopathy are more suggestive features of HIDS. ${ }^{1}$ It may be thought that medical history of the patients is very compatible with HIDS, and FMF should not have to be considered in the working diagnosis of these patients. But it should be remembered that our country has the highest prevalence of FMF worldwide and around $20-26 \%$ of FMF patients have mutation only on one allele. ${ }^{4,5}$ The first case was diagnosed as having FMF or more precisely having one of the periodic fever syndromes and was referred to us because of the recurrence of attacks under colchicine. Interestingly, the second case was diagnosed as FMF by us and initially responded quite well to the colchicine, but later developed typical clinical features of HIDS attacks like rash, diarrhea, cervical lymphadenopathy. The fever duration during attacks was not 5-7 days in every attack in both cases, some attacks were lasting for around 3 days like the attacks of FMF. Fever periods starting during infancy, as it was in both of our cases, might be a clue for HIDS; however, Keskindemirci et al. ${ }^{6}$ reported a 3-month-old girl having FMF with homozygous M694V mutation, which is an example of early onset FMF.

There are some case reports in the literature with digenic mutations in the autoinflammatory disease genes. Digenic inheritance refers to the interaction of two genes resulting in the expression of a phenotype. ${ }^{4}$ Moussa et al. ${ }^{7}$ reported two cases from the same family with overlapping clinical features of FMF and HIDS. Both cases had homozygous mutation in the $M V K$ gene and were compound heterozygous for the $M E F V$ gene. Some attacks of the cases were lasting only 2-3 days and some were 7 days. The authors concluded that having more than one mutation in different genes of monogenic autoinflammatory diseases in the same individual may explain atypical clinical manifestations. Stojanov et al. ${ }^{8}$ reported a case with recurrent fever attacks starting around 1 year of age and lasting for 2-3 weeks every 3 months. While he was 11 years old the attacks started to last 4 days and repeat every 2-4 weeks. Autoinflammatory gene analysis showed that he had novel heterozygous TNFRSF1A gene mutation (Y20D) and heterozygous E148Q variant in the $M E F V$ gene. The authors speculated that heterozygosity for MEFV E148Q variant may serve a disease modifier, resulting in a more severe phenotype of the case. SinghGrewal et al. ${ }^{9}$ presented two independent cases of FMF plus deafness with coexistent $M E F V$ and CIAS1 mutations. Both cases were being followed for splenomegaly, anemia and sensorineural deafness. One at the age of 6 and the other at the age of 8 started to show typical FMF attacks with fever, abdominal pain, arthritis and raised inflammatory markers. Genetic analysis demonstrated that both cases were compound heterozygote for $M E F V$ gene (V726A/E148Q) and one had Q703K and the other had V198M sequence variants in the NLRP3 gene. The authors proposed that synergistic heterozygosity between disease causing mutations in the $M E F V$ gene and nondisease causing or low penetrance sequence variations of the NLRP3 gene may have resulted in the clinical phenotype of FMF with progressive sensorineural deafness as seen in cryopyrin associated periodic syndrome (CAPS). Gülhan et al. ${ }^{10}$ described 2 cases of systemic autoinflammatory diseases that both were having homozygous E148Q mutation in the $M E F V$ gene, but both cases had also unusual findings for FMF such as urticarial rash, nonerysipeloid erythema, lymphadenopathy, and hepatosplenomegaly. Both cases were fulfilling descriptive criteria for FMF, CAPS and systemic juvenile idiopathic arthritis. The cases did not demonstrate any response to colchicine but dramatic response to anakinra. They could not show a second mutation in the MVK, NLRP3 and TNFRSF1A genes and concluded that many other autoinflammatory syndromes might be identified in the future.

In conclusion, for children presenting with symptoms of periodic fever syndromes, more than one diagnosis is possible. Having digenic mutations in the autoinflammatory genes could potentially alter the clinical presentation and response to treatment. We think that, in FMF patients with one or no MEFV mutation that are unresponsive or partially responsive to colchicine or associated with symptoms not typical for FMF, alternative diagnoses 
should be considered and checked for other autoinflammatory gene mutations.

\section{REFERENCES}

1. Barron KS, Kastner DL. Periodic Fever Syndromes and Other Inherited Autoinflammatory Diseases. In: Petty RE, Laxer RM, Lindsey CB, Wedderburn LR (eds). Textbook of Pediatric Rheumatology (7th ed). Philadelphia: Elsevier, 2016: 609-626.

2. Kastner DL, Aksentijevich I, Goldbach-Mansky R. Autoinflammatory disease reloaded: A clinical perspective. Cell 2010; 140: 784-790.

3. Russo RA, Brogan PA. Monogenic autoinflammatory diseases. Rheumatology (Oxford) 2014; 53: 1927-1939.

4. Ozen S. Changing concepts in familial Mediterranean fever: Is it possible to have an autosomal-recessive disease with only one mutation? Arthritis Rheum 2009; 60: 1575-1577.

5. Caso F, Rigante D, Vitale A, et al. Monogenic autoinflammatory syndromes: state of the art on genetic, clinical, and therapeutic issues. Int J Rheumatol 2013; 2013: 513782 .
6. Keskindemirci G, Aktay Ayaz N, Aldemir E, Aydoğmuş C, Aydoğan G, Kavuncuoğlu S. Familial Mediterranean fever: Diagnosing as early as 3 months of age. Case Rep Pediatr 2014; 2014: 296479.

7. Moussa T, Aladbe B, Taha RZ, Remmers EF, El-Shanti H, Fathalla BM. Overlap of familial Mediterranean fever and hyper-IgD syndrome in an Arabic kindred. J Clin Immunol 2015; 35: 249-253.

8. Stojanov S, Lohse P, McDermott MF, et al. Periodic fever due to a novel TNFRSF1A mutation in a heterozygous Chinese carrier of MEFV E148Q. Rheumatology (Oxford) 2004; 43: 526-527.

9. Singh-Grewal D, Chaitow J, Aksentijevich I, Christodoulou J. Coexistent MEFV and CIAS 1 mutations manifesting as familial Mediterranean fever plus deafness. Ann Rheum Dis 2007; 66: 1541.

10. Gülhan B, Büyükcam A, Touitou I, Özen S. Diagnostic dilemma in autoinflammatory disease in two patients: does the name matter? Turk J Pediatr 2013; 55: 315318. 